\title{
In silico study on Penicillin derivatives and Cephalosporins for upper respiratory tract bacterial pathogens
}

\author{
K. M. Kumar $\cdot$ P. Anitha $\cdot$ V. Sivasakthi • \\ Susmita Bag $\cdot$ P. Lavanya $\cdot$ Anand Anbarasu • \\ Sudha Ramaiah
}

Received: 24 August 2012/Accepted: 25 May 2013/Published online: 11 June 2013

(c) The Author(s) 2013. This article is published with open access at Springerlink.com

\begin{abstract}
Upper respiratory tract infection (URTI) is an acute infection which involves the upper respiratory tract: nose, sinuses, tonsils and pharynx. URT infections are caused mainly by pathogenic bacteria like Streptococcus pneumoniae, Haemophilus influenzae and Staphylococcus aureus. Conventionally, $\beta$-lactam antibiotics are used to treat URT infections. Penicillin binding proteins (PBPs) catalyze the cell wall synthesis in bacteria. $\beta$-Lactam antibiotics like Penicillin, Cephalosporins, Carbapenems and Monobactams inhibit bacterial cell wall synthesis by binding with PBPs. Pathogenic bacteria have efficiently evolved to resist these $\beta$-lactam antibiotics. New generation antibiotics are capable of inhibiting the action of PBP due to its new and peculiar structure. New generation antibiotics and Penicillin derivatives are selected in this study and virtually compared on the basis of interaction studies. 3-Dimensional (3D) interaction studies between Lactivicin, Cefuroxime, Cefadroxil, Ceftaroline, Ceftobiprole and Penicillin derivatives with PBPs of the abovementioned bacteria are carried out. The aim of this study was to suggest a potent new generation molecule for further modification to increase the efficacy of the drug for the URTI.
\end{abstract}

Keywords Upper respiratory tract infections . Penicillin binding proteins $-\beta$-Lactam antibiotics . Docking

K. M. Kumar · P. Anitha · V. Sivasakthi · S. Bag ·

P. Lavanya - A. Anbarasu - S. Ramaiah ( $)$

School of Biosciences and Technology, VIT University,

Vellore 632014, Tamil Nadu, India

e-mail: sudhaanand@vit.ac.in

\section{Introduction}

The respiratory tract is a frequent site of infection because it comes in direct contact with the physical environment and is exposed to airborne microorganisms. Worldwide, approximately 4 million children under 5 years of age die each year from respiratory tract infections (RTIs) (Garenne et al. 1992). It is estimated that throughout the world 1.9 million children $<5$ years old died from acute respiratory infection in 2001, 70\% of them in Africa and South East Asia (Williams et al. 2001). Nasopharyngitis, pharyngitis, tonsillitis and otitis media are common upper respiratory tract (URT) infections which constitute $87.5 \%$ of the total episodes of respiratory infections. URT infections can be caused by a variety of bacteria like Chlamydia pneumoniae, Mycoplasma pneumoniae, Streptococcus pyogenes, Streptococcus pneumoniae, Bordetella pertussis, Staphylococcus aureus, Escherichia coli and Haemophilus influenzae (Peter et al. 1985). The majority of URT infections are caused by only three species $S$. pneumoniae, $S$. aureus (Gram-positive bacteria) and $H$. influenzae (Gram-negative bacteria). The treatments of these three bacterial infections have been more complicated by the emergence and spread of multi-drug resistant strains (Doern et al. 1988, 1997). Two mechanisms have been reported to be responsible for antibiotic resistance: structural modification in Penicillin binding protein (PBP) targets and production of $\beta$-lactamase, first identified in 1972 (Williams and Moosdeen 1986; Reid et al. 1987; Jorgensen 1992). PBPs are the membrane bound enzymes which catalyze the steps involved in bacterial cell wall biosynthesis and are the target enzymes of $\beta$-lactam antibiotics (Ghuysen 1991; Goffin and Ghuysen 1998; Macheboeuf et al. 2006; Sauvage et al. 2008). Peptidoglycan is the major component of bacterial cell wall synthesized by PBPs. Every bacterial species has more than two PBPs. 
S. pneumoniae, the major human pathogen causing URT infections is responsible for over 1.6 million deaths every year (Lynch and Zhanel 2005). It has six PBPs, PBP1a, PBP1b, PBP2a, PBP2b, PBP2x and PBP3, which are highly conserved. Penicillin resistance in $S$. pneumoniae has been reported in many countries. The mechanism of Penicillin resistance is due to the modification of active site motif in PBPs of S. pneumoniae. Penicillins and extended spectrum Cephalosporins have high level of resistance to PBP1a, PBP2x and PBP2b of S. pneumoniae (Sheldon and Mason 1998). S. aureus is a potent pathogen that can cause respiratory tract infections (Ragle et al. 2010). It has PBP1b, PBP2a and PBP3. The resistance of $S$. aureus to Penicillin was identified in 1940 and 1965, but recently it has become a major threat to public health concern (Metan et al. 2005), alteration in PBP2a encoded gene decreases the affinity of most $\beta$-lactam antibiotics. $H$. influenzae is a common and exclusively human commensal of the nasopharynx. $\mathrm{H}$. influenzae colonizes in the nasal cavity of approximately $80 \%$ of the human population. H. influenzae has PBP4 and PBP5 which are low molecular weight proteins. The treatment of $H$. influenzae infections has been more complicated by the emergence and spread of multi-drug resistant strains (Doern et al. 1988, 1997). Several computational investigations have been done on $\beta$-lactam antibiotics and PBPs. Yoshida et al. reported the crystal structures of PBP3 in methicillinresistant S. aureus (MRSA) and nature of its interactions with Cefotaxime. The study explains in detail about the hydrophobic and hydrogen bond interaction of Cefotaxime with the active sites of the PBP3 and PBP2 of S. aureus. Experimentally they proved it with nanoelectrospray mass spectrometry and ultracentrifugation to measure its sensitivity to different types of Penicillin derivatives (Yoshida et al. 2012). Samo Turk et al. study mainly focused to discover non-covalent inhibitor for PBP2x and PBP2a experimentally and computationally. The study reported the minimum inhibitory concentration of non-covalent inhibitor against several Gram-positive bacterial strains, including MRSA and analyzed the binding affinity of inhibitor with PBP2a and PBP2x (Turk et al. 2011). Another computational study investigated the interaction of Carbenicillin, Ceftazidime and Cefotaxime with binding site of PBP1b and PBP3 (Sainsbury et al. 2011). Sainsbury et al. reported the crystal structures of apo-PBP and complexes with Ceftazidime and Carbenicillin and investigated the similarities and differences between these structures. Fumihiro Kawai et al. determined the high-resolution apo crystal structures of twolow molecular weight PBPs, PBP4 and PBP5 from $H$. influenzae. They demonstrated the binding affinity of designed $\beta$-lactam antibiotics and Amoxicillin with PBP4 and PBP5 (Kawai et al. 2010). Though Penicillin derivatives and Cephalosporins have been used for bacterial infections over a period of time, many bacterial pathogens have become resistant to these antibiotics. One major mode of resistance is by the alternation of PBPs resulting in low affinity to $\beta$ lactam antibiotics. Researchers have explored the mechanism of resistance to $\beta$-lactam antibiotics using only a few Penicillin derivatives or Cephalosporins (Sainsbury et al. 2011; Turk et al. 2011; Yoshida et al. 2012). This prompted us to investigate in detail using a wide spectrum of $\beta$-lactam antibiotics (both Penicillin derivatives and Cephalosporins). Our results indicate that of $19 \beta$-lactam antibiotics, Ceftobiprole and Ceftaroline might have better affinity to PBPs and hence it may be effective in the treatment of URT bacterial infections. Our results are also comparable to previous experimental findings (Hebeisen et al. 2001; Sader et al. 2005; Jones et al. 2005; Kosowska et al. 2005; Davies et al. 2006; Citron and Goldstein 2008; Estrada et al. 2008; Henry et al. 2010; Kosowska et al. 2010; Mosian et al. 2010; Dauner et al. 2010) and the findings of our research might provide clues as to how Ceftobiprole and Ceftaroline exert their inhibitory action on bacterial pathogens.

\section{Methods}

Preparation of macromolecular and small molecular models

PBP was thought to be essential for the synthesis of bacterial cell wall. All types of the PBPs (PBP1a, PBP1b, PBP2a, PBP2b, PBP2x, PBP3, PBP4, PBP5 and PBP6) were selected for this study. 3-Dimensional (3D) structures of the PBPs were obtained from Protein Data Bank (PDB) (Berman et al. 2000). 3D structures of PBPs were visualized through PyMOL viewer (Lill and Danielson 2010). Co-crystallized ligands were identified and removed from the target proteins then water molecules removed and $\mathrm{H}$ atoms were added to the structure and minimizations were performed using Swiss pdb viewer (Guex and Peitsch 1997). The 3D coordinates of the Penicillin derivatives and Cephalosporins were obtained from NCBI PubChem Compound database (Li et al. 2010) and constructed using chemsketch ( $\mathrm{Li}$ et al. 2004). Hydrogen atoms were added to all the structures and gasteiger atomic partial charges were computed. A geometry optimization of all the compounds was performed using chimera (Pettersen et al. 2004) for flexible conformations of the compounds during the docking.

PDB ID of every PBP was depicted in Table 1 and twodimensional structures of Penicillin derivatives and Cephalosporins are shown in Fig. 1.

Active site identification

The catalytic binding site was believed to be a small region, a cleft or pocket, where lead molecules can bind to 
Table 1 Active site residues of PBPs

\begin{tabular}{llll}
\hline PBPs & PDB ID & Name of the organism & Active site residues \\
\hline PBP1a & 2C6W & Streptococcus pneumoniae & Ala270, Tyr271, Asp273, Asn274, Trp311, Asn315, Leu345, Gly346, Ala347, \\
& & & Arg348, His349, Hln350, Ser351 \\
PBP1b & 2Y2Q & Staphylococcus aureus & Asp337, Phe341, Thr342, Ala345, Glu346, Glu349, Tyr443, Gln447, Asn448, \\
& & & Asn449, Phe452, Asp453, Glu540 \\
PBP2a & 1VQQ & Staphylococcus aureus & Ser403, Lys406, Arg445, Tyr446, Glu447, Ile459, Glu460, Ser403, Ser462, \\
& & & Asp463, Asn464 \\
PBP2b & 2WAE & Streptococcus pneumoniae & Thr55, Thr56, Ser57, Ser81, Gln180, Ala183, Val184, Gly185, Ala188, Thr189, \\
& & & Gly190, Thr191, Ser218, Ser258, Leu259, Asn260, Asp261, Arg 262, Arg280 \\
PBP2x & 1PYY & Streptococcus pneumoniae & Lys420,Val423, Pro424, Thr425, Arg426, Arg463, Glu476, Glu497, Ile498, \\
& & & Val499, Gly500, Ala650, Arg654, Pro660, Ile661, Val662, Gly664 \\
PBP3 & 3OC2 & Streptococcus pneumoniae & Ala162, His163, Gly166, Phe167, Arg175, Glu176, Gly177, Leu180, Tyr268, \\
& & & Pro278, Met281, Arg282, Asn283, Met286, Ile287, Phe383, Pro384, Gly385, \\
& & & Glu386, Arg387 \\
PBP4 & 1TVF & Staphylococcus aureus & Gln133, Val136, Ser137, Asn138, Ser139, Phe225, Phe225, Thr226, Lys227, \\
& & & Gln228, Tyr239, Thr240, Phe241, Asn242, Leu245, Leu258, Lys259, Thr260 \\
PBP5 & 3A3J & Haemophilus influenzae & Val75, Val77, Leu79, Lys80, Asn86, Asn121, Asp193, Leu194, Leu194, Pro195, \\
& & & Glu196, Glu197, Ile200 \\
PBP6 & 3ITB & Escherichia coli & Ser40, Ile103, Ile104, Gln105, Ser106, Pro192, Asn193, Arg194, Asn195, \\
& & & Met208, Lys209, Thr210, Gly211, Thr212
\end{tabular}

stimulate the target protein and produce the desirable effect. Thus, recognizing the catalytic binding site residues in the protein structure was of high importance in computer-aided drug designing. Identification of accurate catalytic binding site was difficult because the target proteins were capable of undergoing conformational changes (Liao and Andrews 2007). Qsite finder (Laurie and Jackson 2005) recognizes the possible ligand binding sites using the van der Waal's probes and interaction energy. In the present study, Qsite finder was employed for locating the active sites in PBP1a, PBP1b, PBP2a, PBP2b, PBP2x, PBP3, PBP4, PBP5 and PBP6 proteins.

Virtual screening of $\beta$-lactam antibiotics

iGEMDOCK (A Generic Evolutionary Method for molecular DOCKing) automated docking program (Yang and Chen 2004). iGEMDOCK integrated the structurebased virtual screening, molecular docking, post screening analysis and visualization steps. We selected all types of PBPs (PBP1a, PBP1b, PBP2a, PBP2b, PBP2x, PBP3, PBP4, PBP5 and PBP6) to carry out the structure-based virtual screening study of penicillin derivatives and Cephalosporins. The 3D coordinates of each therapeutic target protein and ligand molecules were implemented through the GEMDOCK graphical environment interface. Before docking, the output path was set. GEMDOCK default parameters included the population size ( $n=200)$, generation $(g=70)$ and number of solutions ( $s=10$ ) to compute the probable ligand binding mechanism for each target protein. Then the docking run was started using GEMDOCK scoring function. After docking, the individual binding pose of each ligand was observed and their binding affinity with the target proteins was analyzed. In the post docking screening the best binding pose and total energy of each ligand was analyzed. The details of best binding pose and total energy values were saved in output folder. Protein-ligand binding site was analyzed and visualized using PyMOL (Lill and Danielson 2010).

\section{Docking}

The automated docking studies were carried out using Auto-Dock version 4.0 (Morris et al. 2009). 3D structure of each PBPs were implemented through the graphical user interface AUTODOCKTOOLS (ADT 1.4.6). The graphical user interface AUTODOCKTOOLS was performed to set up the enzymes: all hydrogens were added, Kollman United Atoms charges loaded and non-polar hydrogens were merged to carbon atoms. The initial parameters and van der Waals well depth of $0.100 \mathrm{kcal} / \mathrm{mol}$ for macromolecules, generated PDBQT files were saved. The 3D structures of ligand molecules were constructed, optimized, and converted into Mol2 file format with the help of the chimera. The charges of the non-polar hydrogen atoms are assigned to the atom to which the hydrogen is attached. The resulting files were saved as PDBQT files. The drug binding site for the ligands on PBP1a, PBP1b, PBP2a, PBP2b, PBP2x, PBP3, PBP4, PBP5 and PBP6 were identified using Qsite finder online server. The grid point was set at the ligand binding site in each one of the obtained 
(a)

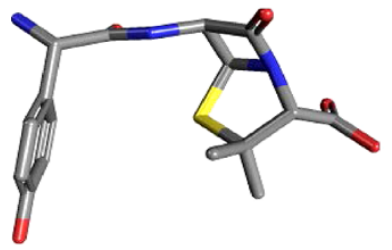

(b)

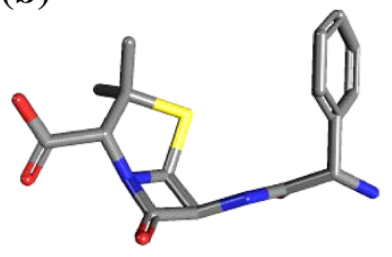

(c)

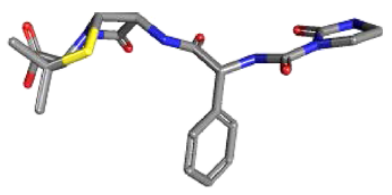

(d)

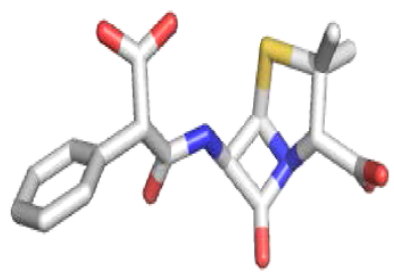

(e)

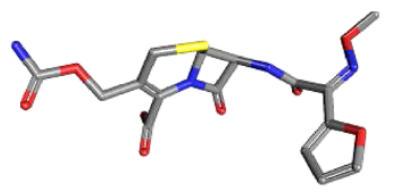

(f)

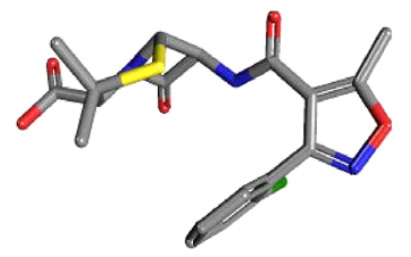

(g)

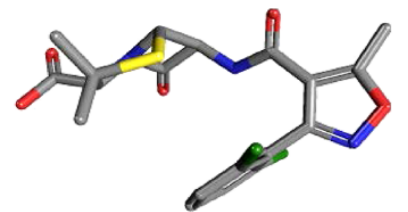

(h)

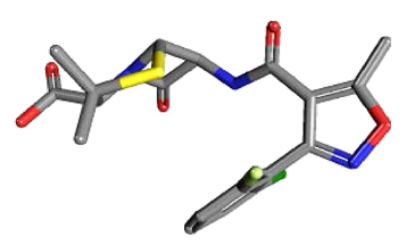

(i)

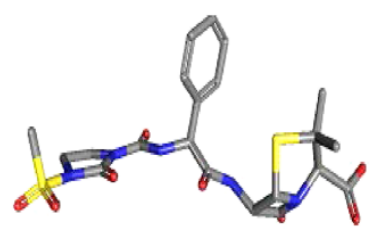

(j)

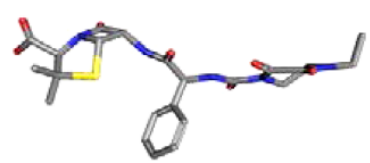

(k)

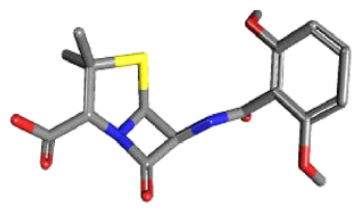

(I)

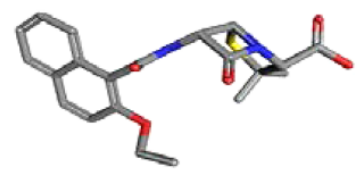

(m)

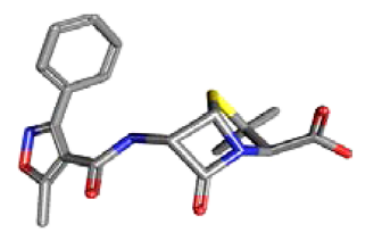

(n)

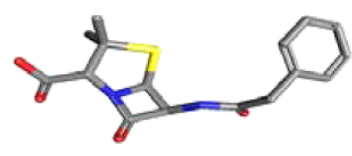

(s)

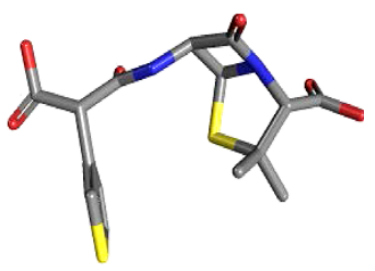

(p)

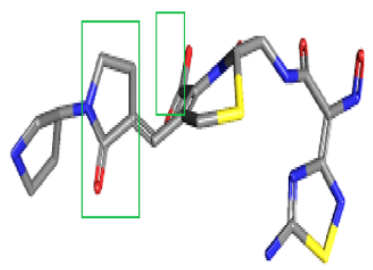

(q)

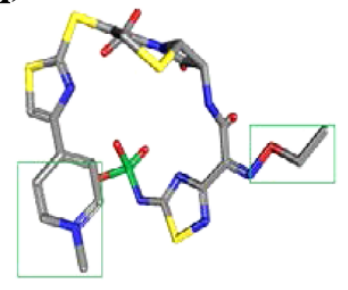

(r)

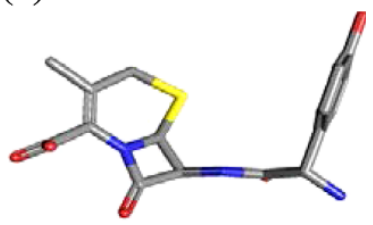

(o)

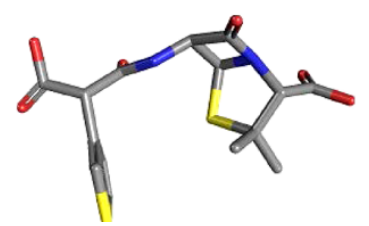

Fig. 1 3-Dimensional structures of Penicillin derivatives and Cephalosporins: a Amoxicillin, b Ampicillin, c Azlocillin, d Carbenicillin, e Cefuroxime, f Cloxacillin, g Dicloxacillin, h Flucloxacillin, i Mezlocillin, j Piperacillin, k Methicillin, I Nafcillin, m Oxacillin,

PDB structures. AUTODOCK 4.0 was performed for all docking calculations. The AUTODOCKTOOLS was used to generate the grid parameter files and docking parameter files. The docking parameters were also used to calculate docking scores for $\beta$-lactam antibiotics and Penicillin derivatives. Protein-ligand docking calculations were carried out on PBPs. Lamarckian genetic algorithm (Morris

n Penicillin G, o Ticarcillin, p Ceftobiprole, q Ceftaroline, $\mathbf{r}$ Cefadroxil and $\mathbf{s}$ Lactivicin (The highlighted boxes indicate the nonessential components in $\mathbf{p}$ Ceftobiprole and $\mathbf{q}$ Ceftaroline respectively)

et al. 1998) was used to generate possible protein-ligand binding conformations.

ADME screening

The molinspiration (Jarrahpour et al. 2011) server was used to predict the ADME properties of the antibiotics. It 
predicted both physiochemical and pharmacological properties. Smiles (Simplified Molecule Input Line Entry Specification) of the antibiotics was submitted. It predicted the properties of the drug such as molecular volume, number of hydrogen bond donors and acceptors, LogP and rotatable bonds. It provided high-speed molecular properties calculated and drug likeness for a given compound. The acceptability of the analogs is evaluated based on Lipinski's rule of 5 (Lipinski et al. 2006), which is essential for structure-based drug design.

\section{Results and discussion}

The 3D structures of PBP1a, PBP1b, PBP2a, PBP2b, PBP2x, PBP3, PBP4, PBP5 and PBP6 are analyzed and 19 $\beta$-lactam antibiotics are optimized to have minimal potential energy using chimera and then the virtual screening study is carried out for ligand molecules. From the virtual screening analysis, we list binding mode of Penicillin derivatives and Cephalosporins based on total energy (Table 2). The best binding poses for each ligand molecule into each target protein are determined and the one having lowest binding energy among the different poses generated. The lower energy scores represent better protein-ligand binding affinity compared to higher energy values. Among the 19 ligands, Cephalosporins are found to have lower binding energy value than the Penicillin derivatives. Especially the fifth generation Cephalosporins, Ceftaroline and Ceftobiprole has least binding energy value. Ceftobiprole shows best binding pose with PBP1b, PBP2a, PBP2b and PBP2x (total energy value for $\mathrm{PBP} 1 \mathrm{~b}=-110.7 \mathrm{kcal} / \mathrm{mol}, \quad$ PBP2a $=-108.2 \mathrm{kcal} / \mathrm{mol}$, $\mathrm{PBP} 2 \mathrm{~b}=-110.4 \mathrm{kcal} / \mathrm{mol}, \quad \mathrm{PBP} 2 \mathrm{x}=-116 \mathrm{kcal} / \mathrm{mol})$. The Ceftaroline shows best binding conformation with PBP3, PBP4, PBP5 and PBP6 (total energy for PBP3 = $-114 \mathrm{kcal} / \mathrm{mol}, \quad$ PBP4 $=-104.8 \mathrm{kcal} / \mathrm{mol}, \quad$ PBP5 $=$ $-131.2 \mathrm{kcal} / \mathrm{mol}$ and $\mathrm{PBP} 6=-118.0 \mathrm{kcal} / \mathrm{mol})$. On comparing the binding mode of Penicillin derivative, Azlocillin shows higher binding affinity with PBP1a (total energy value $=-122.1 \mathrm{kcal} / \mathrm{mol}$ ). These compounds have more stable ligand-receptor complex amongst other compounds. We further analyzed the docked conformation for finding the binding mode of fifth generations Cephalosporins, Ceftaroline and Ceftobiprole into selected target proteins to validate the position obtained likely to represent reasonable binding modes or conformations.

\section{Docking of Ceftobiprole into PBPs}

Docking simulation of Ceftobiprole is performed for PBP1a, PBP1b, PBP2a, PBP2b, PBP2x, PBP3, PBP4, PBP5 and PBP6. From the docking result, we identified that Ceftobiprole has best binding affinity with the PBP2x of S. aureus. Docking of Ceftobiprole results in the formation of more than five hydrogen bonds with PBP1b,

Table 2 Virtual screening results of $\beta$-lactam antibiotics by iGEMDOCK

\begin{tabular}{|c|c|c|c|c|c|c|c|c|c|c|}
\hline S. no & \#Ligand & PBP-1A & PBP-1B & PBP-2A & PBP-2B & PBP-2X & PBP-3 & PBP-4 & PBP-5 & PBP-6 \\
\hline 1 & Amoxicillin & -121.1 & -103.7 & -89.8 & -88.2 & -84.1 & -94.5 & -67.6 & -98.7 & -79.9 \\
\hline 2 & Ampicillin & -89.3 & -69.3 & -87.3 & -76.1 & -82.1 & -86.0 & -64.8 & -94.4 & -84.4 \\
\hline 3 & Azlocillin & -122.1 & -83.3 & -99.8 & -93.9 & -84.4 & -100.4 & -72.9 & -91.4 & -85.0 \\
\hline 4 & Carbenicillin & -86.6 & -74.9 & -91.8 & -84.7 & -97.6 & -98.2 & -75.5 & -107.6 & -90.4 \\
\hline 5 & Cefadroxil & -107.5 & -87.4 & -90.1 & -100.3 & -101.7 & -112.9 & -72.3 & -88.6 & -83.4 \\
\hline 6 & Ceftobiprole & -104.6 & -110.7 & -108.2 & -110.4 & -116.0 & -113.0 & -83.2 & -113.0 & -102.0 \\
\hline 7 & Ceftaroline & -104.3 & -97.1 & -79.4 & -97.0 & -104.4 & -114.1 & -104.8 & -131.2 & -118.0 \\
\hline 8 & Cefuroxime & -103.4 & -104.0 & -94.6 & -110.3 & -94.3 & -86.1 & -78.2 & -91.0 & -80.0 \\
\hline 9 & Cloxacillin & -91.4 & -90.9 & -83.3 & -89.4 & -82.0 & -89.4 & -69.0 & -94.3 & -82.0 \\
\hline 10 & Dicloxacillin & -95.9 & -83.9 & -85.7 & -82.6 & -99.1 & -97.1 & -62.7 & -85.2 & -84.0 \\
\hline 11 & Flucloxacillin & -89.6 & -75.4 & -96.6 & -88.9 & -74.9 & -89.8 & -62.1 & -90.8 & -82.1 \\
\hline 12 & Lactivicin & -91.1 & -95.9 & -87.1 & -90.9 & -95.8 & -95.3 & -65.6 & -98.1 & -89.0 \\
\hline 13 & Methicillin & -95.5 & -102.1 & -102.0 & -97.0 & -92.3 & -109.6 & -74.9 & -93.5 & -94.0 \\
\hline 14 & Mezlocillin & -88.6 & -101.9 & -102.1 & -92.9 & -97.4 & -105.0 & -72.4 & -112.1 & -97.1 \\
\hline 15 & Nafcillin & -89.3 & -100.8 & -77.3 & -82.0 & -111.4 & -101.5 & -67.1 & -97.8 & -84.3 \\
\hline 16 & Oxacillin & -101.6 & -88.5 & -94.2 & -86.9 & -78.1 & -90.3 & -67.5 & -97.4 & -87.1 \\
\hline 17 & Penicillin G & -84.3 & -74.4 & -77.3 & -79.3 & -83.1 & -89.5 & -66.2 & -81.6 & -72.2 \\
\hline 18 & Piperacillin & -89.2 & -86.4 & -81.3 & -97.5 & -97.1 & -103.7 & -73.4 & -99.8 & -88.0 \\
\hline 19 & Ticarcillin & -99.8 & -79.1 & -81.6 & -85 & -80.9 & -86.3 & -64.7 & -95.2 & -92.0 \\
\hline
\end{tabular}

The values in bold font indicate best binding energies 

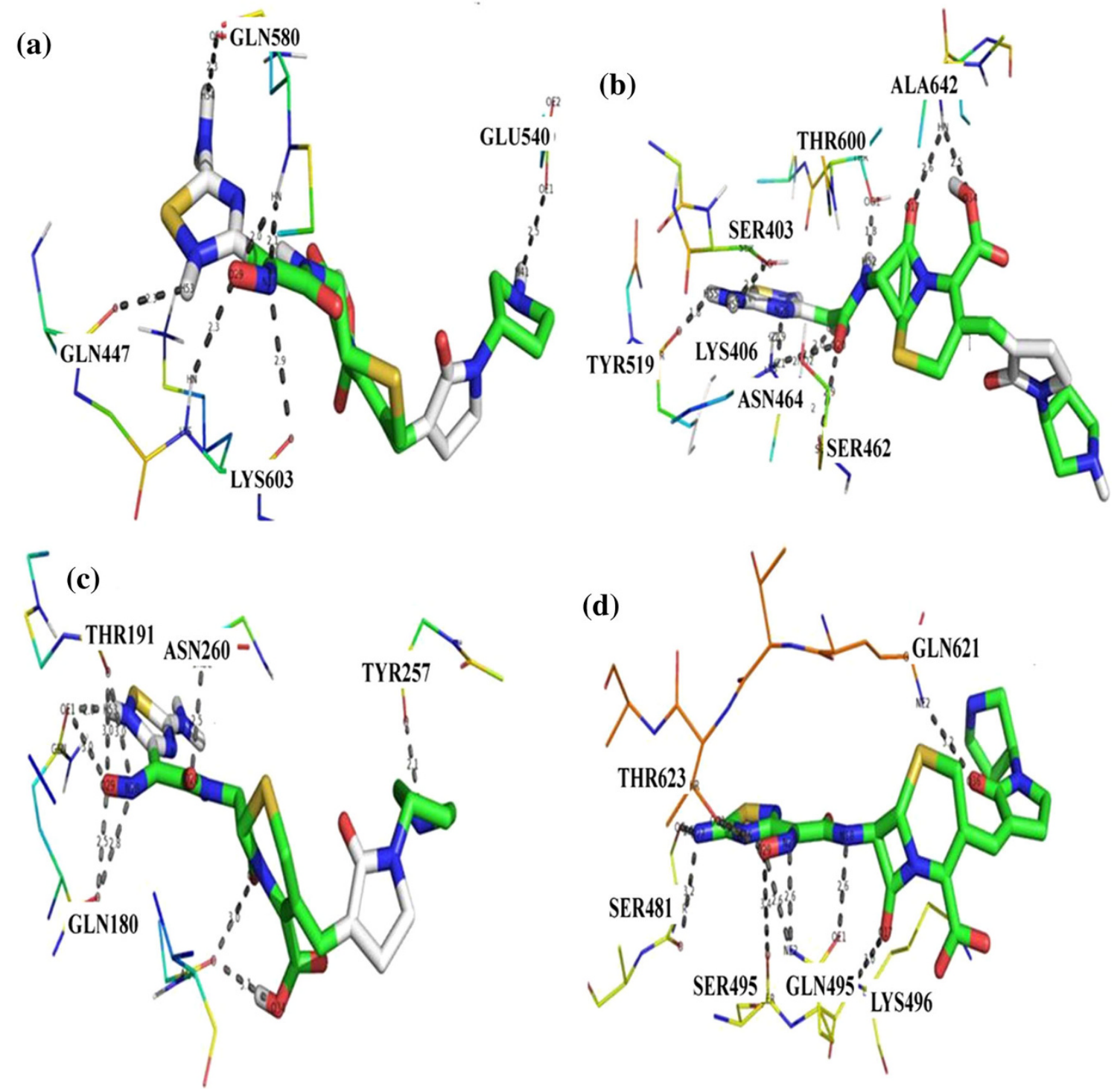

Fig. 2 Docking results of Ceftobiprole against PBP1b, PBP2a, PBP2b and PBP2x. a Binding mode of Ceftobiprole in PBP1b. b A close-up view of the binding site of Ceftobiprole in PBP2a. c Ceftobiprole interaction with PBP2b. d Binding mode of

PBP2a, PBP2b and PBP2x (Fig. 2). Amino acid residues Gln582, Glu540, Lys603 and Gln601 are involved in interaction with PBP1b; in PBP2a, the interacting amino acids are Ala642, Thr600, Tyr519, Ser403, Ser462, Asn464 and Lys406. In PBP2b, Asn260, Tyr257, Thr191 and Gln180 are involved in the interaction with Ceftobiprole. In close assessment of this binding mode, binding docking energies are calculated for PBP1b, PBP2a, PBP2b, and PBP2x (Table 3). In PBP2x, the amino acid residues Gln621, Lys496, Gln495, Ser481 and Thr623 interact with Ceftobiprole (Table 4). Davies et al. (2006) report that Ceftobiprole itself inhibits PBP1a, PBP2b and PBP2x, which are responsible for Penicillin resistance in $S$.
Ceftobiprole with PBP2x. Ligand atoms are colored by its type. The interacted amino acids residues, hydrogen bond networks in the binding pocket and the distance (in $\AA$ units) of bonds are all shown

pneumoniae. Our results are similar to the findings of Davies et al. Ceftobiprole, a fifth generation Cephalosporin in phase 3 clinical trials, exhibits a broad spectrum of activities against many clinically important Gram-positive and Gram-negative pathogens, such as $S$. pneumoniae, $H$. influenzae, and S. aureus (Hebeisen et al. 2001; Jones et al. 2002; Kosowska et al. 2005; Zbinden et al. 2002). Docking analysis of Ceftobiprole shows best results against $S$. pneumoniae and $S$. aureus. Our results are similar to previous studies (Hebeisen et al. 2001; Jones et al. 2002; Kosowska et al. 2005). Lovering et al. (2012) report that the affinity of Ceftobiprole to PBP2a of MRSA is high. Henry et al. (2010) 
report that PBP5 has less sensitivity to Ceftobiprole than PBP2a. Another study reveals that Ceftobiprole is a novel broad-spectrum antibiotic that inhibits PBP2a and

Table 3 AutoDock estimated docked energies of Ceftobiprole and Ceftaroline

\begin{tabular}{llll}
\hline S. no & Target & $\begin{array}{l}\text { Ceftobiprole } \\
(\mathrm{kcal} / \mathrm{mol})\end{array}$ & $\begin{array}{l}\text { Ceftaroline } \\
(\mathrm{kcal} / \mathrm{mol})\end{array}$ \\
\hline 1 & PBP1a & -5.1 & -5.2 \\
2 & PBP1b & -6.76 & -4.12 \\
3 & PBP2a & -6.12 & -3.43 \\
4 & PBP2b & -7.04 & -5.1 \\
5 & PBP2x & -7.32 & -5.3 \\
6 & PBP3 & -6.1 & -7.42 \\
7 & PBP4 & -4.34 & -5.65 \\
8 & PBP5 & -6.21 & -9.2 \\
9 & PBP6 & -5.3 & -8.3 \\
\hline
\end{tabular}

Table $4 \mathrm{H}$-bond interactions and bond length obtained for Ceftobiprole with PBP1b, PBP2a, PBP2b and PBP2x

\begin{tabular}{|c|c|c|}
\hline Protein-ligand complex & H-bond interactions & Bond length $(\AA)$ \\
\hline \multirow[t]{6}{*}{ Ceftobiprole-PBP1b } & (Gln 582)O-H54 & 2.3 \\
\hline & (Gln 582)NH-029 & 2.0 \\
\hline & (Gln 582)NH-N28 & 2.1 \\
\hline & (Glu 540)O-H41 & 2.5 \\
\hline & (Lys 603)O-N28 & 2.9 \\
\hline & (Lys603)NH-O29 & 2.3 \\
\hline \multirow[t]{8}{*}{ Ceftobiprole-PBP2a } & (Ala642)NH-O34 & 2.5 \\
\hline & (Ala642)NH-O17 & 2.6 \\
\hline & (Thr600)O-H52 & 1.8 \\
\hline & (Tyr 519)O-H55 & 1.8 \\
\hline & (Ser403)O-H54 & 2.1 \\
\hline & $(\mathrm{Ser} 462) \mathrm{O}-\mathrm{O} 20$ & 2.9 \\
\hline & (Asn 464)H-O29 & 2.0 \\
\hline & (Lys 406)H-O20 & 2.8 \\
\hline \multirow[t]{6}{*}{ Ceftobiprole-PBP2b } & $($ Asn260)H-O20 & 2.5 \\
\hline & (Tyr257)O-H41 & 2.1 \\
\hline & (Thr191)O-O29 & 3.0 \\
\hline & (Thr191)O-N28 & 3.0 \\
\hline & $(\mathrm{Gln} 180) \mathrm{O}-\mathrm{O} 29$ & 3.0 \\
\hline & $(\mathrm{Gln} 180) \mathrm{O}-\mathrm{N} 28$ & 2.8 \\
\hline \multirow[t]{8}{*}{ Ceftabiprole-PBP2x } & $(\mathrm{Gln} 621) \mathrm{N}-\mathrm{O} 36$ & 3.2 \\
\hline & (Lys496)N-O17 & 3.0 \\
\hline & $(\mathrm{Gln} 495) \mathrm{O}-\mathrm{N} 18$ & 2.6 \\
\hline & $(\mathrm{Gln} 495) \mathrm{N}-\mathrm{N} 28$ & 2.8 \\
\hline & $(\mathrm{Gln} 180) \mathrm{N}-\mathrm{O} 29$ & 2.6 \\
\hline & (Ser 495)O-O29 & 3.4 \\
\hline & (Thr 623)O-O29 & 3.2 \\
\hline & (Ser 481)O-N27 & 3.2 \\
\hline
\end{tabular}

PBP2x, which are responsible for the resistance in $S$. pneumoniae and $S$. aureus, respectively (Dauner et al. 2010). Though many reports on the inhibitory activity of Ceftobiprole for specific PBPs are available in literature, none of the studies have focused on the binding pattern of Ceftobiprole to all type of PBPs. Our study reveals the binding pattern of Ceftobiprole with all type of PBPs. The possible binding mode of Ceftobiprole in the PBP1b, PBP2a, PBP2b, PBP2x binding site and corresponding 2D interaction models along with hydrogen bonds and bond distance are shown in Fig. 2.

\section{Docking of Ceftaroline into PBPs}

Ceftaroline is a antibiotic of the Cephalosporin type among the majority of currently available $\beta$-lactam antibiotics. Cephalosporins are used for effective treatment of bacterial respiratory tract infections. In our results on the binding conformation modes of Penicillin derivatives and Cephalosporins with PBPs, Ceftaroline shows higher affinity with the PBP3, PBP4, PBP5 and PBP6 than the other PBPs. In examining the interaction and position of the Ceftaroline in PBP3, PBP4, PBP5 and PBP6 active site predicted by our docking procedure, it is observed that multiple hydrogen

Table 5 H-bond interactions and bond length obtained for Ceftaroline with PBP3, PBP4, PBP5 and PBP6

\begin{tabular}{|c|c|c|}
\hline Protein-ligand complex & H-bond interactions & Bond length $(\AA)$ \\
\hline \multirow[t]{4}{*}{ Ceftaroline-PBP3 } & (Arg54)NH-O15 & 2.7 \\
\hline & $(\mathrm{Gln} 121) \mathrm{N}-\mathrm{O} 13$ & 3.2 \\
\hline & (Tyr124)OH-O43 & 1.8 \\
\hline & (Tyr124)O-O23 & 3.2 \\
\hline Ceftaroline-PBP4 & (Asn260)O-N18 & 3.1 \\
\hline \multirow[t]{6}{*}{ Ceftaroline-PBP5 } & (Ala311)N-O42 & 3.1 \\
\hline & $(\mathrm{G} \ln 366) \mathrm{N}-\mathrm{N} 30$ & 3.1 \\
\hline & (Phe312)N-O43 & 2.7 \\
\hline & $(\operatorname{Arg} 192) \mathrm{N}-\mathrm{O} 15$ & 2.8 \\
\hline & $(A s n 47) \mathrm{N}-\mathrm{O} 13$ & 2.6 \\
\hline & $(\mathrm{Asn} 47) \mathrm{O}-\mathrm{N} 11$ & 3.1 \\
\hline \multirow[t]{11}{*}{ Ceftaroline-PBP6 } & (Thr270)N-O14 & 3.0 \\
\hline & (Thr270)O-O14 & 2.7 \\
\hline & (Arg194)NH-O43 & 3.2 \\
\hline & (Arg194)NH-O23 & 3.2 \\
\hline & (Asn193)N-N10 & 3.4 \\
\hline & (Asn193)O-N11 & 3.1 \\
\hline & (Asn193)O-O13 & 2.6 \\
\hline & (Ile104)O-N11 & 2.7 \\
\hline & (Met208)O-O13 & 3.5 \\
\hline & (Lys209)N-O15 & 3.0 \\
\hline & (Ser106)O-O5 & 3.1 \\
\hline
\end{tabular}



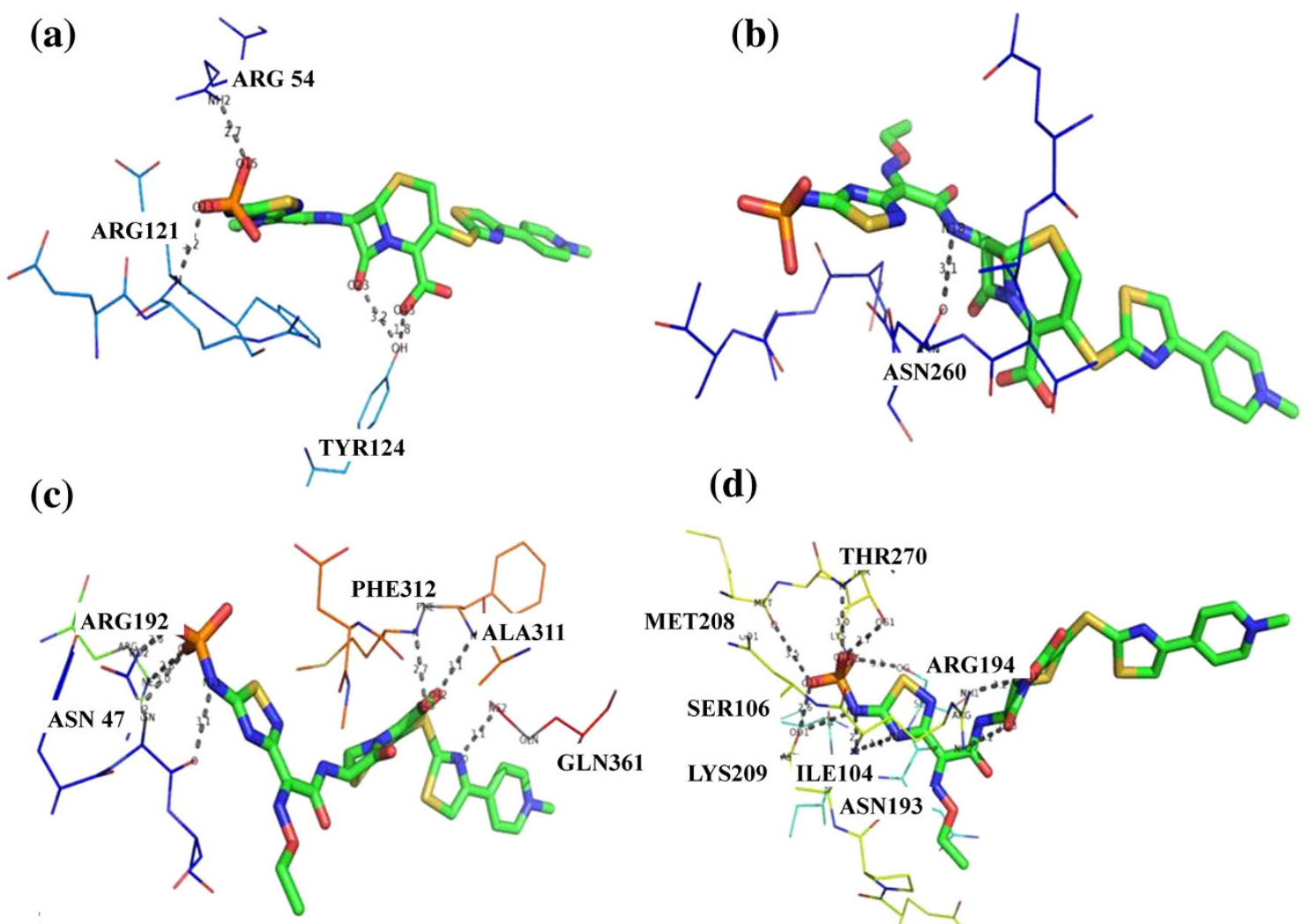

(d)

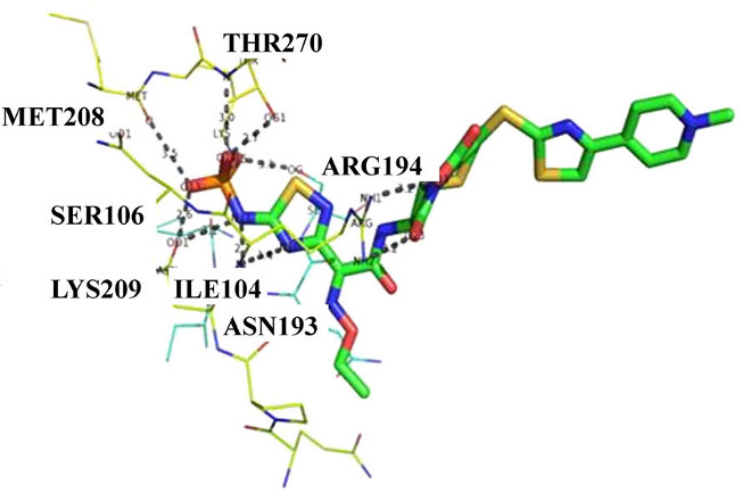

Fig. 3 Docked complex of Ceftaroline-PBP3, PBP4, PBP5 and PBP6. a A close-up view of the predicted binding site for Ceftaroline in PBP3. b Binding mode of Ceftaroline with PBP4. c Ceftaroline binding site in PBP5. (3D) Interaction of Ceftaroline with PBP6.

bonds are formed (Table 5). In addition, the amino acid residues Arg54, Glu121 and Tyr124 of PBP3 are involved in van der Waals' interactions. In PBP4, only one amino acid residue Asn260 is involved in interaction with Ceftaroline. Binding of Ceftaroline to PBP5 and PBP6 involves more than six hydrogen bonds. The binding affinity of Ceftaroline for MRSA PBP2a, methicillin-susceptible $S$. aureus (MSSA) PBPs 1 to 3, and $S$. pneumoniae $\mathrm{PBP} 2 \mathrm{x} / 2 \mathrm{a} / 2 \mathrm{~b}$ correlates well with its low MICs and bactericidal activity against these resistant organisms (Kosowska et al. 2010; Moisan et al. 2010). Citron et al. report the effects of Ceftaroline activity against Gram-positive and Gram-negative pathogens, including MSSA, MRSA, E. faecalis, S. pyogenes, $S$. pneumoniae, $H$. influenzae, $M$. catarrhalis, K. pneumonia, E. coli, $P$. aeruginosa, and $A$. baumannii (Citron and Goldstein 2008; Jones et al. 2005). Other studies reveal that Ceftaroline has potent activity against MRSA and S. pneumoniae. The Gram-negative spectrum of Ceftaroline is similar to that of other broadspectrum Cephalosporins (Estrada et al. 2008; Moisan et al. 2010; Kosowska et al. 2010). Morrissey et al. report that the Ceftaroline has excellent activity against MRSA and

Ligand atoms are colored by its type. The interacted amino acids residues, hydrogen bond networks in the binding pocket and the distance (in $\AA$ units) of bonds are all shown

Penicillin-resistant S. pneumoniae. Furthermore, Ceftaroline maintains good activity against $H$. inlfuenzae (Sader et al. 2005; Mushtaq et al. 2007; Morrissey et al. 2009). Our results are consistent with the previously studied ones (Kosowska et al. 2010; Moisan et al. 2010; Citron and Goldstein 2008; Jones et al. 2005; Estrada et al. 2008; Kosowska et al. 2010; Sader et al. 2005; Mushtaq et al. 2007; Morrissey et al. 2009). Although many studies have been reported the inhibitory action of Ceftaroline to specific PBPs, no studies have been done for the binding pattern of Ceftaroline with all type of PBPs. Our results clearly explain the binding pattern of Ceftaroline with all type of PBPs. The binding energy calculated by AutoDock for Ceftaroline-PBP complexes is shown in Table 3. The best possible binding mode of Ceftaroline in PBP4, PBP5 and PBP6 and their corresponding 2D interaction models are displayed in Fig. 3.

\section{ADME screening}

For each of the Penicillin derivatives and Cephalosporins, we analyzed for a number of physiochemical properties 
and pharmaceutically relevant properties, such as molecular weight, H-bond donors, H-bond acceptors, $\log \mathrm{P}$ (octanol/water), and their position according to Lipinski's rule of 5 (Table 6). Lipinski's rule of 5 is a rule of thumb to predict drug likeness, or determine if a compound with a certain biological or pharmacological activity has properties that would make it a likely orally active drug in humans. The rule describes physiochemical properties important for a drug's pharmacokinetics in the human body, including its ADME. The drug molecule shows poor absorption and permeation when they have more than 5 hydrogen bond donors, molecular weight over $500, \log \mathrm{P}$ is over 5 and more than 10 hydrogen bond acceptors. In this study, of the 19 ligands, 16 structures showed possible values for the properties analyzed and exhibited drug-like characteristics based on Lipinski's rule of 5. Methicillin has more than 7 rotatable bonds. Rotatable bond more than 10 and molecular weight more than 500 can lead to decreased permeability and oral bioavailability. But Ceftobiprole and Ceftaroline show molecular weight more than 500. Hence to improve the action of these two drugs, we have highlighted the non-essential regions (Fig. 1) that may possibly be spliced to reduce the molecular mass. However, the effectiveness of these low molecular mass compounds has to be tested in both in vivo and in vitro.

Table 6 Molecular properties of Penicillin derivatives and Cephalosporins obtained from Molinspiration

\begin{tabular}{llclrlc}
\hline $\begin{array}{l}\text { S. } \\
\text { no }\end{array}$ & Antibiotics & $\begin{array}{l}\text { LogP } \\
(<5)\end{array}$ & $\begin{array}{l}\text { Molecular } \\
\text { weight } \\
(<500 \\
\text { dalton })\end{array}$ & $\begin{array}{l}\text { HBA } \\
\text { count } \\
(<10)\end{array}$ & $\begin{array}{l}\text { HBD } \\
\text { count } \\
(<5)\end{array}$ & $\begin{array}{l}\text { Rotatable } \\
\text { bond } \\
\text { count } \\
(<7)\end{array}$ \\
\hline 1 & Amoxicillin & 2.31 & 365.40 & 6 & 4 & 4 \\
2 & Ampicillin & -2.00 & 349.40 & 5 & 3 & 4 \\
3 & Azlocillin & 0.20 & 461.49 & 6 & 4 & 5 \\
4 & Carbenicillin & 1.13 & 378.39 & 6 & 3 & 5 \\
5 & Cloxacillin & 2.61 & 435.88 & 5 & 2 & 4 \\
6 & Dicloxacillin & 2.90 & 470.32 & 5 & 2 & 3 \\
7 & Flucloxacillin & 2.69 & 453.87 & 5 & 2 & 3 \\
8 & Methicillin & 0.85 & 380.41 & 10 & 3 & 11 \\
9 & Mezlocillin & 0.21 & 539.58 & 8 & 3 & 5 \\
10 & Nafcillin & 3.21 & 414.47 & 5 & 2 & 5 \\
11 & Oxacillin & 2.05 & 401.43 & 5 & 2 & 4 \\
12 & Penicillin G & 1.5 & 334.39 & 4 & 2 & 4 \\
13 & Piperacillin & 1.2 & 517.55 & 7 & 2 & 6 \\
14 & Ticarcillin & 0.99 & 384.42 & 6 & 3 & 5 \\
15 & Ceftobiprole & -1.68 & 564.16 & 11 & 7 & 4 \\
16 & Ceftaroline & 2.43 & 699.03 & 16 & 5 & 2 \\
17 & Cefadroxil & -1.22 & 377.10 & 7 & 5 & 3 \\
18 & Lactivicin & -0.60 & 296.14 & 5 & 1 & 2 \\
19 & Cefuroxime & -0.2 & 424.39 & 10 & 3 & 7 \\
\hline & & & & & & \\
\hline
\end{tabular}

\section{Conclusion}

In the present study, molecular docking studies were performed to explore possible binding modes of Penicillin derivatives and Cephalosporins into all types of PBPs, PBP1a, PBP2b, PBP2x and PBP3 of $S$. pneumoniae, PBP1b, PBP2a and PBP4 of S. aureus, PBP5 of H. influenzae, as these organisms are most frequently found pathogens in the URT. The molecular docking study revealed that the Cephalosporins show higher affinity with PBPs than the Penicillin derivatives. Especially the fifth generation Cephalosporins, Ceftobiprole and Ceftaroline show best results to all types of PBPs. The binding affinity was evaluated by the binding free energies (DGb, Kcal/ mol) and hydrogen bonding. The compounds which revealed the highest binding affinity are the ones with lowest binding free energy. On comparing the binding energy and the binding site residues, we found that all compounds differ in their binding modes or binding site residues for hydrogen bond formation. The conclusion drawn from this virtual screening and docking result was that the Ceftobiprole has highest binding affinity with the PBP2x of $S$. pneumoniae. The Ceftaroline has maximum number of interaction with PBP5 of $H$. influenzae. The above results suggest that the Ceftobiprole and Ceftaroline can be potent inhibitors for all types of PBPs. From ADME screening of all the 19 compounds, 16 compounds satisfied Lipinski's rule of 5. Ceftobiprole and Ceftaroline show molecular weight more than 500 which decreases their permeability and bioavailability. These drugs can further be modified to satisfy Lipinski's rule of 5. Though, there are a few reports on the in vitro analysis of Ceftobiprole and Ceftaroline, there are no in silico studies that predict the binding and active regions in these molecules. Our study is probably the first such attempt and we infer that our results will throw light for the future development of more potent next generation antibiotics for the treatment of upper respiratory infections and counter the emergence of antibiotic resistant strains.

Acknowledgments Dr. Anand Anbarasu gratefully acknowledges the Indian council of Medical Research (ICMR), Government of India Agency for the research grant [IRIS ID: 2011-03260]. P. Lavanya thanks ICMR for the Research fellowship through the ICMR grant [IRIS ID: 2011-03260]. The authors would also like to thank the management of VIT University for providing the necessary facilities to carry out this research project.

Conflict of interest The authors declare that there is no conflict of interest.

Open Access This article is distributed under the terms of the Creative Commons Attribution License which permits any use, distribution, and reproduction in any medium, provided the original author(s) and the source are credited. 


\section{References}

Berman HM, Westbrook J, Feng Z, Gilliland G, Bhat TN, Weissig H, Shindyalov IN, Bourne PE (2000) The protein data bank. Nucleic Acids Res 28:235-242

Citron DM, Goldstein EJC (2008) Effects of in vitro test method variables on ceftaroline activity against aerobic Gram-positive and Gram-negative pathogens, poster D-2232. In: Interscience Conference on Antimicrobial Agents and Chemotherapy. Infectious Disease Society of America. American Society for Microbiology, Washington, DC

Dauner DG, Nelson RE, Taketa DC (2010) Ceftobiprole: a novel, broad-spectrum cephalosporin with activity against methicillinresistant Staphylococcus aureus. Am J Health Sys Pharm 67(12):983-993

Davies TA, Shang W, Bush K (2006) Activities of Ceftobiprole and other $\beta$-lactams against Streptococcus pneumoniae clinical isolates from the United States with defined substitutions in penicillin-binding proteins $\mathrm{PBP} 1 \mathrm{a}, \mathrm{PBP} 2 \mathrm{~b}$, and PBP 2x. Antimicrob Agents Chemother 50:2530-2532

Doern GV, Jorgensen JH, Thornsberry C (1988) National collaborative study of the prevalence of antimicrobial resistance among clinical isolates of Haemophilus influenzae. Antimicrob Agent Chemother 32:180-185

Doern GV, Brueggmann AB, Pierce G, Holley HP, Rauch A (1997) Antibiotic resistance among clinical isolates of in the United States in 1994 and 1995 and detection of $\beta$-lactamase-positive strains resistant to amoxicillin-clavulanate: results of a national multicenter surveillance. Antimicrob Agents Chemother 41:292-297

Estrada VA, Lee M, Hesek D, Vakulenko SB, Mobashery S (2008) Co-opting the cell wall in fighting methicillin-resistant Staphylococcus aureus: potent inhibition of PBP 2a by two anti-MRSA b-lactam antibiotics. J Am Chem Soc 130:9212-9213

Garenne M, Ronsmans C, Campbell H (1992) The magnitude of mortality from acute respiratory infections in children under 5 years in developing countries. World Health Stat Q 45:180-191

Ghuysen JM (1991) Serine $\beta$-lactamases and Penicillin-binding proteins. Annu Rev Microbiol 45:37-67

Goffin C, Ghuysen JM (1998) Multimodular Penicillin-binding proteins: an enigmatic family of orthologs and paralogs. Microbiol Mol Biol Rev 62:1079-1093

Guex N, Peitsch MC (1997) SWISS-MODEL and the SwissPdbViewer: an environment for comparative protein modeling. Electrophoresis 18:2714-2723

Hebeisen PI, Krauss H, Angehrn P, Hohl P, Page MGP, Then RL (2001) In vitro and in vivo properties of Ro 63-9141, a novel broad-spectrum cephalosporin with activity against methicillinresistant staphylococci. Antimicrob Agents Chemother 45: $825-836$

Henry X, Amoroso A, Coyette J, Joris B (2010) Interaction of Ceftobiprole with the low-affinity PBP 5 of Enterococcus faecium Antimicrob. Agents Chemother 54:953955

Jarrahpour A, Fathi J, Mimouni M, Benhadda T, Sheikh J, Chohan ZH, Petra AP (2011) Osiris and molinspiration (POM) together as a successful support in drug design: antibacterial activity and biopharmaceutical characterization of some azo schiff bases. Med Chem Res 19(7):1-7

Jones RN, Deshpande LM, Mutnick AH, Biedenbach BJ (2002) In vitro evaluation of BAL9141, a novel parenteral cephalosporin active against oxacillin-resistant staphylococci. J Antimicrob Chemother 50:915-932

Jones RN, Fritsche TR, Ge Y, Kaniga K, Sader HS (2005) Evaluation of PPI-0903 M (T91825), a novel cephalosporin: bactericidal activity, effects of modifying in vitro testing parameters and optimization of disc diffusion tests. J Antimicrob Chemother 56:1047-1052

Jorgensen JH (1992) Update on mechanisms and prevalence of antimicrobial resistance in Haemophilus influenzae. Clin Infect Dis 14:1119-1123

Kawai F, Clarke TB, Roper D, Han GJ, Hwang KY, Unzai S, Obayashi E, Park SY, Tame RH (2010) Crystal Structures of Penicillin-Binding Proteins 4 and 5 from Haemophilus Influenzae. J Mol Biol 396:634-645

Kosowska SK, Hoellman DB, Lin G, Clark C, Credito K, McGhee P, Dewasse B, Bozdogan B, Shapiro S, Appelbaum PC (2005) Anti pneumococcal activity of ceftobiprole, a novel broad-spectrum cephalosporin. Antimicrob Agents Chemother 49:1932-1942

Kosowska SK, McGhee PL, Appelbaum PC (2010) Affinity of ceftaroline and other $\beta$-lactams for penicillin-binding proteins from Staphylococcus aureus and Streptococcus pneumoniae. Antimicrob Agents Chemother 54:1670-1677

Laurie A, Jackson R (2005) Q-SiteFinder: an energy-based method for the prediction of protein-ligand binding sites. Bioinformatics 21:1908-1916

Li Z, Wan H, Shi Y, Ouyang P (2004) Personal experience with four kinds of chemical structure drawing software: review on ChemDraw, ChemWindow, ISIS/Draw, and ChemSketch. J Chem Inf Comput Sci 44:1886-1890

Li Q, Cheng T, Wang Y, Bryant SH (2010) PubChem as a public resource for drug discovery. Drug Discov Today 15(23-24): 1052-1057. doi:101016/jdrudis201010003

Liao JJ, Andrews RC (2007) Targeting protein multiple conformations: a Structure-based strategy for kinase drug design. Curr Top Med Chem 7:1394-1407

Lill MA, Danielson ML (2010) Computer-aided drug design platform using PyMOL. J Comput Aided Mol Des 25:13-19

Lipinski CA, Lombardo F, Dominy PW, Feeney PJ (2006) Experimental and computational approaches to estimate solubility and permeability in drug discovery and development settings. Adv Drug Deliv Rev 23:3-25

Lovering AL, Gretes MC, Safadi SS, Danel F, Castro LD, Page MGP, Strynadka NCG (2012) Structural insights into the antimethicillin-resistant Staphylococcus aureus (MRSA) activity of ceftobiprole. J Biol Chem 287:32096-32102

Lynch JP, Zhanel GG (2005) Escalation of antimicrobial resistance among Streptococcus pneumoniae: implications for therapy. Semin Respir Crit Care Med. 26:575-616

Macheboeuf P, Martel CC, Job V, Dideberg O, Dessen A (2006) Penicillin binding proteins: key players in bacterial cell cycle and drug resistance processes. FEMS Microbiol Rev 30:673-691

Metan G, Zarakolu P, Unal S (2005) Rapid detection of antibacterial resistance in emerging Gram-positive cocci. J Hosp Infect 61:93-99

Moisan H, Pruneau M, Malouin F (2010) Binding of Ceftaroline to penicillin binding proteins of Staphylococcus aureus and Streptococcus pneumoniae. J Antimicrob Chemother 65:713-716

Morris GM, Goodsell DS, Halliday RS, Huey R, Hart WE, Belew RK, Olson AJ (1998) Automated docking using a Lamarckian genetic algorithm and an empirical binding free energy function. J Comput Chem 19:1639-1662

Morris GM, Huey R, Lindstrom W, Sanner MF, Belew RK, Goodsell DS, Olson AJ (2009) AutoDock4 and AutoDockTools4: automated docking with selective receptor flexibility. J Comput Chem 30:2785-2791

Morrissey I, Ge Y, Janes R (2009) Activity of the new cephalosporin Ceftaroline against bacteraemia isolates from patients with community-acquired pneumonia. Int $\mathrm{J}$ Antimicrob Agents 33(6):515-519 
Mushtaq S, Warner M, Ge Y, Kaniga K, Livermore DM (2007) In vitro activity of Ceftaroline (PPI-0903 M, T-91825) against bacteria with defined resistance mechanisms and phenotypes. J Antimicrob Chemother 60:300-311

Peter E, Derek RF, Brown J (1985) Penicillin-binding proteins of $\beta$ lactam-resistant strains of Staphylococcus aureus. FEBS Lett 192:28-32. doi:10.1016/0014-5793

Pettersen EF, Goddard TD, Huang CC, Couch GS, Greenblatt DM (2004) UCSF Chimera -a visualization system for exploratory research and analysis. J Comput Chem 25:1605-1612

Ragle BE, Karginov VA, Wardenburg JB (2010) Prevention and treatment of Staphylococcus aureus with a $\beta$-cyclodextrin derivative. Antimicrob Agents Chemother 54(1):298-304

Reid AJ, Simpson IN, Harper PB, Amyes SG (1987) Ampicillin resistance in Haemophilus influenzae: identification of resistance mechanisms. J Antimicrob Chemother 20:645-656

Sader HS, Fritsche TR, Kaniga K, Jones RN (2005) Antimicrobial activity and spectrum of PPI-0903 M (T-91825), a novel cephalosporin, tested against a worldwide collection of clinical strains. Antimicrob Agents Chemother 49:3501-3512

Sainsbury S, Bird L, Rao V, Sharon M, Shepherd, Stuart DI, Hunter WN, Owens RJ, Ren J (2011) Crystal structures of Penicillinbinding protein 3 from Pseudomonas aeruginosa: comparison of native and antibiotic-bound forms. J Mol Biol 405:173-184

Sauvage E, Kerff F, Terrak M, Ayala JA, Charlier P (2008) The Penicillin-binding proteins: structure and role in peptidoglycan biosynthesis. FEMS Microbiol Rev 32:234-258
Sheldon LK, Mason OE (1998) Management of infections due to antibiotic-resistant Streptococcus pneumoniae. Clin Microbiol Rev 11:628-644

Turk S, Verlaine O, Gerards T, Zivec M, Humljan J, Sosic I, Amoroso A, Zervosen A, Luxen A, Joris B, Gobec S (2011) New noncovalent Inhibitors of Penicillin-Binding Proteins from Penicillin-Resistant Bacteria. Plos one 6:5-e19418

Williams JD, Moosdeen F (1986) Antibiotic resistance in: epidemiology, mechanisms and therapeutic possibilities. Rev Infect Dis 8(Suppl 5):S555-S561

Williams BG, Gouws E, Boschi-Pinto C, Bryce J, Dye C (2001) Estimates of world-wide distribution of child deaths from acute respiratory infections. Lancet Infect Dis 2(1):25-32

Yang M, Chen CC (2004) GEMDOCK: a generic evolutionary method for molecular docking. Protein Structure Funct Bioinfo 55:288-304

Yoshida H, Kawai F, Obayashi E, Akashi S, Roper DI, Tame JR, Park SY (2012) Crystal structures of penicillin-binding protein 3 (PBP3) from methicillin-resistant staphylococcus aureus in the apo and cefotaxime bound forms. J Mol Biol 423:351-364

Zbinden R, Punter V, Graevenitz AV (2002) In vitro activities of BAL9141, a novel broad-spectrum pyrrolidinone cephalosporin, against Gram-negative non fermenters. Antimicrob Agents Chemother 46:871-874 